\title{
Prevalence of intestinal parasites in companion dogs with diarrhea in Beijing, China, and genetic characteristics of Giardia and Cryptosporidium species
}

\author{
Zhongjia Yu $^{1}$ - Yang Ruan ${ }^{1} \cdot$ Mengjie Zhou ${ }^{1} \cdot$ Siyuan Chen ${ }^{1} \cdot$ Yinxin Zhang ${ }^{1}$.

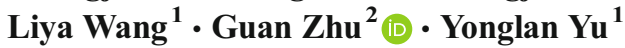

Received: 6 August 2017 / Accepted: 20 September 2017 /Published online: 18 November 2017

(C) Springer-Verlag GmbH Germany 2017

\begin{abstract}
Companion animals including dogs are one of the important components in One Health. Parasites may cause not only diseases in pet animals but also many zoonotic diseases infecting humans. In this study, we performed a survey of intestinal parasites in fecal specimens $(n=485)$ collected from outpatient pet dogs with diarrhea in Beijing, China, for the entire year of 2015 by microscopic examination (all parasites) and SSU rRNA-based nested PCR detection (Giardia and Cryptosporidium). We observed a total of 124 (25.6\%) parasite-positive specimens that contained one or more parasites, including Giardia duodenalis (12.8\%), Cryptosporidium spp. (4.9\%), Cystoisospora spp. (4.3\%), trichomonads (4.3\%), Toxocara canis $(3.5 \%)$, Trichuris vulpis $(0.6 \%)$, and Dipylidium caninum (0.2\%). Among the 55 dog breeds, infection rates were significantly higher in border collies and bulldogs, but lower in poodles $(p<0.05)$. Risk factor analysis suggested that age was negatively correlated with the infection rate $(p<0.00001)$, while vaccination and deworming in the past 12 months could significantly reduce the parasite infections $(p<0.01)$. Among the 62 Giardia-positive specimens, 21 were successfully assigned into assemblages using glutamate dehydrogenase $(g d h)$ and/or beta-giardin $(b g)$ genes, including assemblage $\mathrm{D}(n=15), \mathrm{C}(n=5)$, and $\mathrm{F}(n=1)$. Among the 24
\end{abstract}

Guan Zhu

gzhu@cvm.tamu.edu

Yonglan Yu

gaodifeihao@cau.edu.cn

1 College of Veterinary Medicine, China Agricultural University, Haidian District, Beijing 100193, China

2 Department of Veterinary Pathobiology, College of Veterinary Medicine and Biomedical Sciences, Texas A\&M University, College Station, TX 77843-4467, USA
Cryptosporidium-positive specimens by SSU rRNA PCR, 20 PCR amplicons could be sequenced and identified as Cryptosporidium canis $(n=20)$. Collectively, this study indicates that parasites are a significant group of pathogens in companion dogs in Beijing, and companion dogs may potentially transmit certain zoonotic parasites to humans, particularly those with weak or weakened immunity.

Keywords Companion dogs · Beijing · Giardia duodenalis . Cryptosporidium canis · Cystoisospora spp. Toxocara canis . Trichomonads $\cdot$ Risk factors

\section{Introduction}

Dogs and cats are the most popular species of companion animals (pets) and play an important part in our daily lives. Companion animals are also an important "One Health" component, in which they not only provide many physiological, psychological, and social benefits to humans, but also serve as a reservoir for zoonotic diseases (Matchock 2015; Takashima and Day 2014). Furthermore, a number of parasite species can cause mild to severe diseases in dogs (Robertson and Thompson 2002). Many parasites carried by pet dogs may be transmittable to humans, including cestodes (e.g., echinococcosis), nemotodes (e.g., toxocariosis, dirofilariosis and onchocerciasis), and protozoa (e.g., leishmaniosis, giardiasis and cryptosporidiosis) (Baneth et al. 2016; Gracio et al. 2015; Grinnage-Pulley et al. 2016; Tasic-Otasevic et al. 2015). Additionally, people with immature, weakened, and/or compromised immune systems (e.g., infants, elderly, AIDS patients, and organ transplantation recipients) are susceptible to infections with a wider range of parasitic species, such as Cryptosporidium canis that typically do not infect immunocompetent humans (Adamu et al. 2014; Cama et al. 2007). 
China is home to many companion animals including dogs. Based on a report by Euromonitor International in 2015, China has become the third largest pet market with at least 27 million pet dogs (http://www.euromonitor.com/pet-care-inchina/report). Beijing, the nation's capital, has the largest populations of both human residents (e.g., 21.7 million in 2015 ) and companion animals (e.g., > 0.95 million registered pet dogs in 2012 based on the Beijing Municipal Public Security Bureau; http:/www.bjgaj.gov.cn/web/detail_ getArticleInfo_360104_col1159.html). Despite the importance of companion animals in One Health, there have been a limited number of epidemiological surveys on the zoonotic parasitic diseases in companion dogs in China and virtually no reported data for dogs in Beijing.

Here, we report a year-long survey of gastrointestinal parasites from companion dogs with diarrhea that were brought to the China Agricultural University Veterinary Teaching Hospital from various regions in Beijing in 2015. In the study, we estimated the prevalence of helminths and protozoan parasites. We also genotyped Giardia duodenalis (syn. G. intestinalis, G. lamblia). Our data provided a snapshot of the current prevalence of common intestinal parasites in pet dogs in Beijing that allowed us to discuss their implications in animal well-being and public health.

\section{Materials and methods}

\section{Ethics statement}

This study was performed in strict accordance to the recommendations of the Guide for the Care and Use of Laboratory Animals of the Ministry of Health, China. The research protocol was reviewed and approved by the Research Ethics Committee of the China Agricultural University. Prior to fecal specimen collection, permission was obtained from the animals' owners. All data were de-identified with the removal of private information associated with individual animals.

\section{Sample collection}

Only fresh fecal samples were collected from rectums with catheters or from the excrements of outpatient companion dogs with diarrhea from January 01 to December 31, 2015. Animals were brought to the China Agricultural University Veterinary Teaching Hospital in Beijing by owners seeking medical attention. A total of 485 fecal specimens were collected from dogs representing 16 different administrative districts of Beijing in the north China $\left(39^{\circ} 26^{\prime} \mathrm{N}-41^{\circ} 03^{\prime} \mathrm{N}, 115^{\circ} 25^{\prime} \mathrm{E}\right.$ $\left.-117^{\circ} 30^{\prime} \mathrm{E}\right)$. A questionnaire form was given to each dog owner to collect information on the age, gender, breed, origin, presence or absence of other pet animals, source of food, drinking water, vaccination history and drug treatment (deworming) history within the past 12 month.

Fecal smears (wet mounts) were immediately prepared from freshly collected samples after thoroughly mixing. Smears were examined microscopically for the presence of common parasites by observing eggs of helminthes (e.g., Toxocara canis [canine ascariasis] and Trichuris vulpis [canine whipworm]) and oocysts or trophozoites of protozoa (e.g., coccidia, Giardia duodenalis and trichomonads Tritrichomonas foetus/Pentatrichomonas hominis). The remaining fecal samples were stored at $4{ }^{\circ} \mathrm{C}$ for up to 4 weeks or $-20^{\circ} \mathrm{C}$ for long-term storage without any antibiotics. Total DNA was isolated from fresh or stored fecal samples as described below.

\section{PCR detection and genotyping of $G$. duodenalis and Cryptosporidium spp.}

All fecal samples were washed with PBS once by centrifugation $(3000 \times g$ for $5 \mathrm{~min})$. For each specimen, total genomic DNA was isolated from $200 \mathrm{mg}$ solid material using an E.Z.N.A.R Stool DNA Kit according to the manufacturer's protocol (Omega Biotek Inc., Norcross, GA) (Qi et al. 2015). Fecal DNA was eluted into $50 \mu \mathrm{L}$ DNA wash buffer and stored at $-20{ }^{\circ} \mathrm{C}$. PCR reactions used high-GC SuperStar PCR Mix with Loading Dye or Taq PCR StarMix with Loading Dye (both from GenStar Biosolutions, Beijing) for G. duodenalis genes or Cryptosporidium genes, respectively, as described below.

Molecular detections were carried out by nested PCR amplification of SSU rRNA genes, followed by sequencing to confirm sequence identity. Detection of $G$. duodenalis used previously reported protocols and primers Gia2029 (5'-AAG TGT GGT GCA GAC GGA CTC-3') and Gia2150c (5'-CTG CTG CCG TCC TTG GAT GT-3') in primary PCR, and RH11 (5'-CAT CCG GTC GAT CCT GCC-3') and RH4 (5'-AGT CGA ACC CTG ATT CTC CGC CCA GG-3') in secondary PCR (product size $\sim 290$ bp) (Wang et al. 2014). Detection of Cryptosporidium species used previously described protocols and primers SSU-F2 (5'-TTC TAG AGC TAA TAC ATG CG$\left.3^{\prime}\right)$ and SSU-R2 (5'-CCC ATT TCC TTC GAA ACA GGA$\left.3^{\prime}\right)$ in primary PCR, and SSU-F3 (5'-GGA AGG GTT GTA TTT ATT AGA TAA AG-3') and SSU-R4 (5'-CTC ATA AGG TGC TGA AGG AGT A-3') (product size 840 bp) (Xiao et al. 1999).

Second, genotyping analysis was carried out for specimens positive for $G$. duodenalis based on glutamate dehydrogenase ( $g d h)$ and beta-giardin ( $b g$ ) genes for determining their assemblages. A fragment of Giardia gdh gene was amplified by semi-nested PCR using previously reported protocols and primers GDHeF1 (5'-TCA ACG TYA AYC GYG GYT TCC GT-3') and GDHiR2 (5'-GTT RTC CTT GCA CAT CTC C-3') in primary PCR; and GDHiF3 (5'-CAG TAC 
AAC TCY GCT CTC GG-3') and GDHiR2 in secondary PCR (product size $\sim 430 \mathrm{bp}$ ) (Read et al. 2004). A fragment of Giardia bg gene was amplified by nested PCR using reported protocols and primers G7 (5'-AAG CCC GAC GAC CTC ACC CGC AGT GC-3') and G759 (5'-GAG GCC GCC CTG GAT CTT CGA GAC GAC-3') in primary PCR, and P3 (5'-GAA CGA ACG AGA TCG AGG TCC G-3') and P4 (5'-CTC GAC GAG CTT CGT GTT-3') in secondary PCR (product size $\sim 510 \mathrm{bp}$ ) (Lalle et al. 2005).

All primary PCR reactions were performed in $25 \mu \mathrm{L}$ reaction buffer containing $2 \mu \mathrm{L}$ sample DNA and $100 \mu \mathrm{M}$ each of the primers, while secondary PCR reactions were carried out in $50 \mu \mathrm{L}$ reaction buffer containing $1 \mu \mathrm{L}$ of primary PCR product and $100 \mu \mathrm{M}$ each of the primers. PCR products were analyzed by $1 \%$ agarose gel electrophoresis. Each set of reactions included three controls: a negative reagent control using elution buffer only, a negative sample control using DNA isolated from a pooled fecal samples from dogs previously determined to be negative of both Giardia and Cryptosporidium, and a positive sample control using DNA from pooled fecal samples positive of Giardia or Cryptosporidium. All samples were tested at least twice or until consistent results were obtained. After agarose gel electrophoresis, reactions containing positive PCR products were submitted to Beijing Sunbiotech for isolating DNA from agarose gels and sequencing by Sanger sequencing method using Thermofisher's BigDye Terminator v3.1 cycling sequencing kit and ABI377 DNA sequencer. Each sample was confirmed by bidirectional sequencing and chromatographs for all sequences were manually examined to confirm the accuracy of base calls.

There are six representative (consensus) SSU rRNA nucleotide sequences obtained in this study. These sequences are available in the GenBank, EMBL and DDBJ database under the following accession numbers: KX858906 (representing $n=12$ cases in this study) and KX858907 $(n=6)$ for C. canis; KX858910 $(n=25), \mathrm{KX} 858911(n=23)$, KX858912 $(n=2)$, and KX858913 $(n=3)$ for $G$. duodenalis. Sequences obtained in genotyping analysis were also available in the databases under the accession numbers KY753390 to KY753404 for G. duodenalis gdh and KY753405 to KY753424 for Giardia duodenalis bg genes.

\section{Statistical analysis}

Statistical significances of infection rates between protozoa and helminths and between major dog breeds were evaluated by Fisher's exact test (two-tailed). In risk factor analysis, the odds ratio (OR) for the specified group was calculated in reference to the other group when only two groups were present (e.g., genders) or to the sum of all other groups when more than two groups were present (i.e., seasons). The $95 \%$ confidence intervals $\left(\mathrm{CI}_{95}\right)$ for the ORs were calculated using
Woolf method. The agreement between microscopic examination and PCR detection on $G$. duodenalis was tested by inter-rater reliability Kappa test.

\section{Results and discussion}

\section{Prevalence of intestinal parasites in companion dogs in Beijing with diarrhea}

We collected a total of 485 fecal specimens in year 2015 from outpatient dogs with diarrhea, in which 124 specimens were parasite-positive by wet mount microscopic examination and/ or PCR detection (i.e., an overall positive rate at $25.6 \%$, $n=124$ ) (Table 1, Fig. 1a). There were 2.4-fold more protozoan-positive specimens than the helminths-positive ones (i.e., $19.8 \%, n=96$ vs. $8.2 \%, n=40 ; p<0.0001$ ) including 12 specimens co-infected by protozoa and helminths (Fig. 2a). It should be noted that the actual infection rates for the parasites examined by microscopic examination of fecal smears were most likely higher than those observed due to the low sensitivity of the method, but the results provided a good snapshot on the current status of these parasites in companion dogs in Beijing.

Detected parasites could be classified into seven major groups, including four protozoan groups and three helminth groups: (1) G. duodenalis was the most prevalent parasite in outpatient dogs $(12.8 \%, n=62)$. This parasite was detected using both microscopic and PCR methods. A sample was considered positive when either one or both of the two methods produced positive detection. Between the two methods, nested PCR displayed better sensitivity in detection $(11.1 \%, n=54)$ than microscopy $(8.7 \%, n=42)$. There were 34 specimens with positive detections by both methods $(7.0 \%$, $n=34$ ), whereas there were 20 and 8 specimens that were either PCR-positive but microscopic-negative or microscopic-positive but PCR negative, respectively. Kappa inter-rater agreement test indicated that the "strength of agreement" between the two methods was "good" (Kappa $=0.677$, $\mathrm{SE}= \pm 0.057)$. (2) Cryptosporidium species were detected by nested PCR amplification of SSU rRNA gene. It represented the second most prevalent parasite group $(4.9 \%, n=24)$. (3) Coccidia (Cystoisospora spp.) was the third most prevalent group (4.3\%, $n=21)$. All observed coccidian oocysts belonged to Cystoisospora species, but they were not identified at species level. (4) Trichomonads $(4.3 \%, \mathrm{n}=21)$ were observed but not determined at genus/species level. They were most likely Pentatrichomonas hominis or Tritrichomonas foetus. (5) Ascarids (3.5\%, $n=17)$ were all Toxocara canis based on egg morphology and measurement. (6) Whipworm (Trihuris vulpis) was observed in 3 dog (0.6\%). (7) A single case of tapeworm belonging to Dipylidium caninum was observed in this study $(0.2 \%, n=1)$. Among them, the positive 
Table 1 Summary of infection and co-infection rates by the seven major parasite groups in dogs (total sample $n=485$ )

\begin{tabular}{|c|c|c|c|c|c|c|c|c|}
\hline \multirow[t]{3}{*}{ Parasite groups ${ }^{\mathrm{a}}$} & \multicolumn{2}{|c|}{ Parasite-positive specimens } & \multicolumn{6}{|c|}{ Co-infection by parasite groups } \\
\hline & & & \multicolumn{2}{|c|}{ By 1 parasite group } & \multicolumn{2}{|c|}{ By 2 parasite groups } & \multicolumn{2}{|c|}{ By 3 parasite groups } \\
\hline & $n$ & Rate $\left(\mathrm{CI}_{95}\right), \%$ & $n$ & Rate $\left(\mathrm{CI}_{95}\right), \%$ & $n$ & Rate $\left(\mathrm{CI}_{95}\right), \%$ & $n$ & Rate $\left(\mathrm{CI}_{95}\right), \%$ \\
\hline Giardia duodenalis ${ }^{\mathrm{b}}$ & 62 & $12.8(9.9-16.1)$ & 43 & $8.9(6.5-11.8)$ & 17 & $3.5(2.1-5.6)$ & 2 & $0.4(0-1.5)$ \\
\hline Cryptosporidium spp. ${ }^{\mathrm{c}}$ & 24 & $4.9(3.2-7.3)$ & 15 & $3.1(1.7-5)$ & 8 & $1.6(0.7-3.2)$ & 1 & $0.2(0-1.1)$ \\
\hline Coccidia $^{\mathrm{d}}$ & 21 & $4.3(2.7-6.5)$ & 17 & $3.5(2.1-5.6)$ & 3 & $0.6(0.1-1.8)$ & 1 & $0.2(0-1.1)$ \\
\hline Trichomonads ${ }^{\mathrm{d}}$ & 21 & $4.3(2.7-6.5)$ & 15 & $3.1(1.7-5)$ & 4 & $0.8(0.2-2.1)$ & 2 & $0.4(0-1.5)$ \\
\hline Ascarids $^{\mathrm{d}}$ & 17 & $3.5(2.1-5.6)$ & 9 & $1.9(0.9-3.5)$ & 6 & $1.2(0.5-2.7)$ & 2 & $0.4(0-1.5)$ \\
\hline Whipworm $^{\mathrm{d}}$ & 3 & $0.6(0.1-1.8)$ & 2 & $0.4(0-1.5)$ & 0 & $0(0-0.8)$ & 1 & $0.2(0-1.1)$ \\
\hline Tapeworm $^{\mathrm{d}}$ & 1 & $0.2(0-1.1)$ & 1 & $0.2(0-1.1)$ & 0 & $0(0-0.8)$ & 0 & $0(0-0.8)$ \\
\hline Total protozoa & 96 & $19.8(16.3-23.6)$ & 75 & $15.5(12.4-19)$ & 19 & $3.9(2.4-6.1)$ & 2 & $0.4(0-1.5)$ \\
\hline Total helminths & 40 & $8.2(6-11.1)$ & 27 & $5.6(3.7-8)$ & 10 & $2.1(1-3.8)$ & 3 & $0.6(0.1-1.8)$ \\
\hline Overall infection & 124 & $25.6(21.7-29.7)$ & 102 & $21(17.5-24.9)$ & 19 & $3.9(2.4-6.1)$ & 3 & $0.6(0.1-1.8)$ \\
\hline
\end{tabular}

${ }^{a}$ Total and overall infection rates are smaller than the sums of individual parasite groups because of the presence of co-infections (also see Fig. 2)

${ }^{b}$ Based on PCR detection of SSU rRNA genes and microscopic examination of wet mount slides that were positive by at least one test

${ }^{\mathrm{c}}$ Based on PCR detection of SSU rRNA genes only

${ }^{\mathrm{d}}$ Based on wet mount microscopic examination only. $\mathrm{CI}_{95}=95 \%$ confidence interval

rate of $G$. duodenalis was statistically significantly higher than those of other six parasite groups $(p<0.0001$ by Fisher's exact test), while those of whipworm and tapeworm were significantly lower than the other five groups $(p<0.05$ or better) (Fig. 1a). However, there were no significant differences between cryptosporidial, coccidia, trichomonads, and ascarids ( $p>0.05$ for all possible pairs). Again, Giardia and Cryptosporidium were detected by PCR (vs. others by microscopic examination). The comparison of infection rates between these two protozoa and other parasites was not completely fair but provides a rough idea on the status of parasites in the companion dogs in Beijing.

\section{Prevalence of parasites and dog breeds}

The 485 outpatient dogs comprised of 54 breeds (total $n=450$ ) plus some with mixed breed backgrounds that were grouped together in subsequent analysis $(n=35)$. Among them, the poodle was the most popular breed $(n=142)$, followed by golden retriever $(n=32)$, German shepherd and Siberian husky (each $n=25)$, Labrador retriever $(n=24)$, Pomeranian $(n=21)$, Samoyed $(n=18)$, border collie $(n=15)$, bulldog, chowchow and Pembroke Welsh corgi (each $n=13)$ and Chihuahua $(n=12)$. The case numbers of all other breeds were between nine to one. Considering the power of statistics, statistical analysis was performed for the breeds with 12 or more cases (including those with mixed background as a single category), in which the overall infection rates ranged from $7.7 \%$ (chowchow) to $60.0 \%$ (border collie) (Fig. 1b, Table 2). Fisher's exact test was employed to evaluate whether the positive rate of one specific breed differed significantly from that of the remaining breeds (i.e., the total rate after excluding the breed being tested), in which border collie and bulldog displayed significantly higher positive rates $(p<0.01$ and $p<0.05$ by Fisher's exact test, respectively), and the poodle showed significantly lower positive rates $(p<0.01)$ (Fig. 1b, Table 2). For individual parasite groups, border collie had significantly higher positive rates for Giardia (33.3\%, $p<0.01)$ and Cryptosporidium $(20.0 \%, p<0.05)$. Bulldog and the group of mixed breed background had significantly higher rates for trichomonads $(30.8 \%, p<0.01)$ and ascarids $(14.3 \%, p<0.01)$, respectively. Poodles had significantly lower positive rate for trichomonads $(0.7 \%, p<0.01)$ and ascarids $(0 \%, p<0.05)$ (Table 2).

\section{Risk factor analysis}

Associations between parasite infection rates and nine categories of risk factors acquired from questionnaire forms (e.g., age, origin of dogs, living with other pets, food and drinking water sources, vaccinations and deworming) and clinic records (gender and season) were tested using Fisher's exact test. Among the risk factors, age displayed significant negative correlation with parasite infections (Table 3 ). The chance to carry intestinal parasites was significantly higher in $\leq 6$ months old dogs (overall infection rate $=39.9 \%$, $\mathrm{OR}=5.0, p<0.0001$ ), whereas the chance was significantly lower in $\geq 12$ months old $\operatorname{dogs}$ (rate $=8.3 \%$, OR $=0.6$, $p<0.0001$; vs. rate $=19.0 \%$ in the $6-12$ months age group) (Table 3). This observation is in agreement with the general 

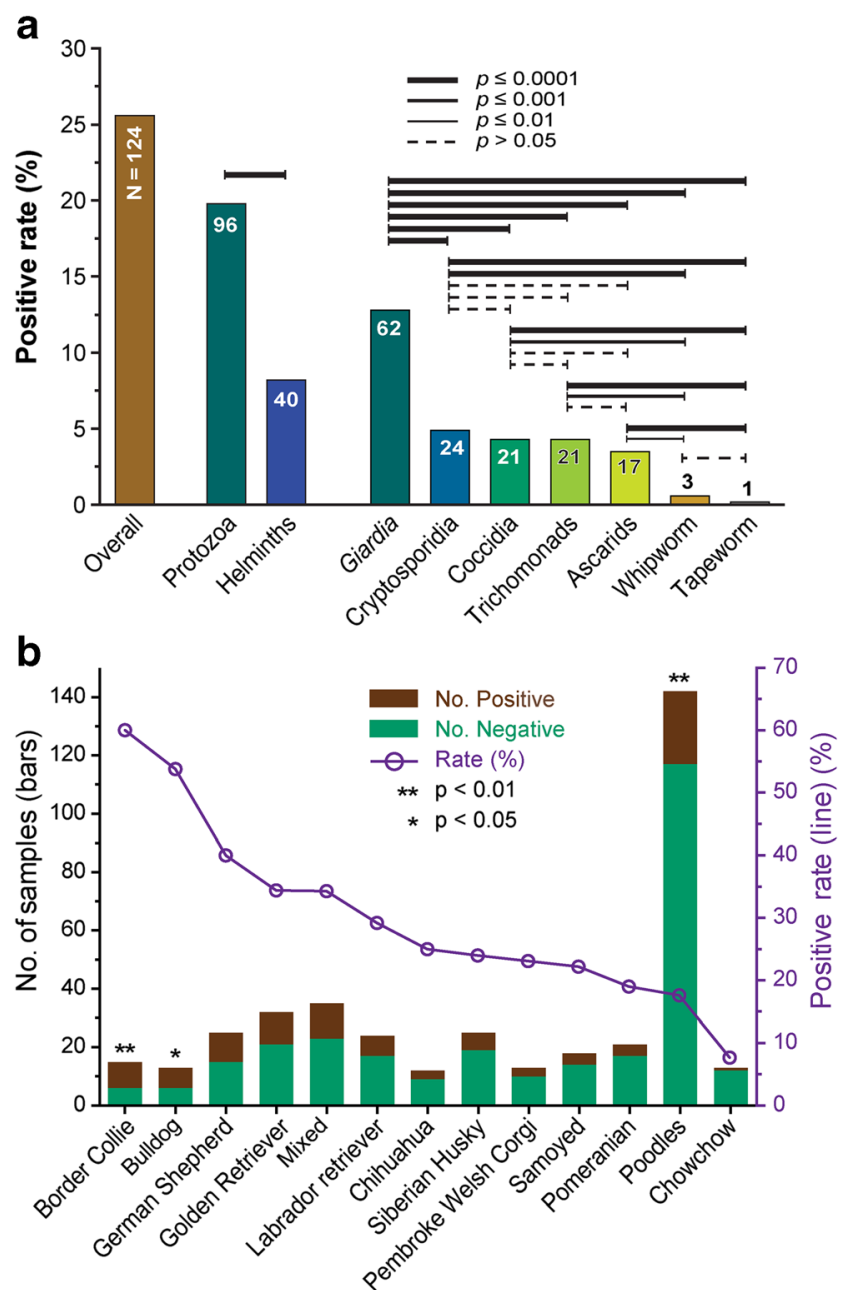

Fig. 1 Summary of parasite infections in companion dogs in Beijing and statistical significance. a Overall infection rate and those by individual parasite groups. b Infection rates by dog breeds. Only breeds with sample sizes $\geq 12$ were used in this analysis. Statistical significance was calculated by two-tailed Fisher's exact test for individual pairs (a) or for individual breeds in reference to the sum of other groups (b). ${ }^{*} p<0.05$; $* * p<0.01 ; * * * p<0.001$

concept that infant and young animals are more susceptible to parasite infections before their immune systems and/or adaptive immunity become fully developed (Abe et al. 2002; Petersen 1992).

Negative correlation was observed between parasite infections and vaccination administrated in the past 12 months before hospital visits $(20.8 \%$ in vaccinated, $\mathrm{OR}=0.5, p<0.01$; vs. $32.8 \%$ in non-vaccinated) (Table 3). Protection from parasite infections by vaccination was statistically significant, although less expected because the vaccines were originally designated for protecting dogs from certain viral and bacterial pathogens, i.e., vaccination by either one or more of the following vaccines: Nobivac DHPPi (canine distemper virus, herpes I, canine parvovirus and canine parainfluenza virus; MSD animal health, New Zealand], Nobivac DHPPi + a

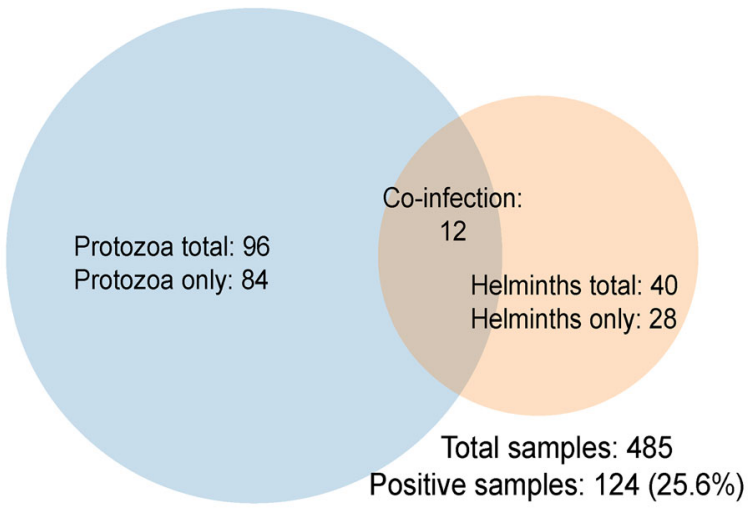

b

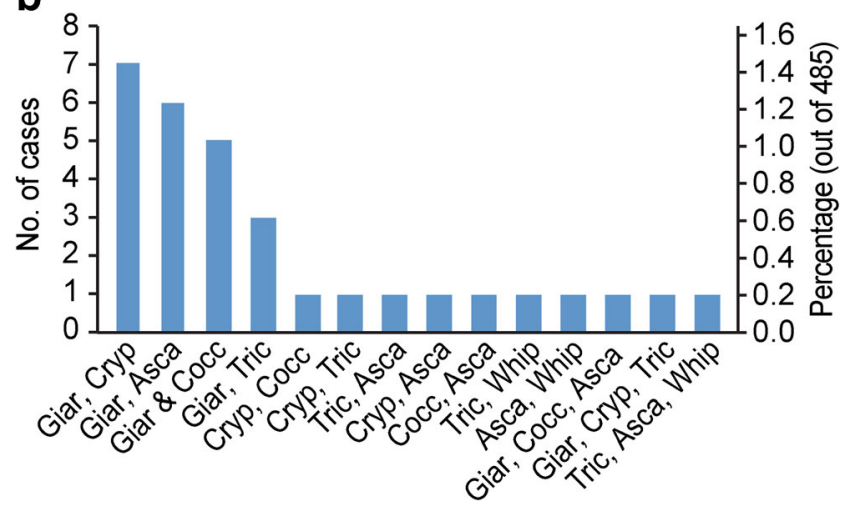

Fig. 2 Parasite co-infection analysis. a Proportional Venn diagram on the co-infections between protozoan and helminths. b Bar chart summary of all co-infected specimens. Giar: Giardia duodenalis, Cryp: Cryptosporidium spp., Asca: Ascarids (Toxocara canis), Tric: trichomonads, Whip: whipworm (Trihuris vulpis)

Lepto (Leptospira canicola and L. icterohemorrhaiae] (MSD animal health), Vanguard plus 5 (canine distemper virus, herpes I/II, canine parvovirus, canine parainfluenza virus) (Zoetis, USA), or Vanguard plus 5/CV-1 (L. canicola, L. icterohemorrhaiae and canine coronavirus) (Zoetis). We speculate that vaccinations significantly reduced (if not fully eliminated) the potential negative effect of common viral infections on the immunity status of dogs to flight parasite infections as postulated in the case of coinfection of parvovirus and Cryptosporidium in a puppy (Denholm et al. 2001). It is also likely that pet owners who had their dogs vaccinated might tend to follow veterinarian's instructions more closely on hygiene practice.

As expected, we also observed significantly less parasite infections in dewormed dogs (rate $=18.6 \%, \mathrm{OR}=0.4$, $p<0.001$; vs. $33.6 \%$ in non-dewormed dogs) (Table 3). Further analysis revealed that deworming reduced the infections by both protozoa and helminths. Statistical significance was observed in the reduction of protozoan infection (rate $=13.6 \%, \mathrm{OR}=0.4, p<0.001$; vs. $26.8 \%$ in non-vaccinated) and not in the reduction of helminth infection (rate $=6.4 \%, \mathrm{OR}=0.6, p=0.1355 ;$ vs. $10.5 \%$ in non- 
Table 2 Positive rates of five major enteric parasite groups in 13 dog breeds

\begin{tabular}{|c|c|c|c|c|c|c|c|c|c|c|c|c|c|}
\hline \multirow[t]{2}{*}{ Breed } & \multirow{2}{*}{$\begin{array}{l}\text { Total } \\
N\end{array}$} & \multicolumn{2}{|c|}{ Overall positivity } & \multicolumn{2}{|c|}{ Giardia duodenalis } & \multicolumn{2}{|c|}{ Cryptosporidium spp. } & \multicolumn{2}{|c|}{ Coccidia } & \multicolumn{2}{|c|}{ Trichomonads } & \multicolumn{2}{|c|}{ Ascarids } \\
\hline & & $N$ & $\%$ & $N$ & $\%$ & $N$ & $\%$ & $N$ & $\%$ & $N$ & $\%$ & $N$ & $\%$ \\
\hline Border collie & 15 & 9 & $60.0 * *$ & 5 & $33.3 *$ & 3 & $20.0 *$ & 0 & 0.0 & 2 & 13.3 & 0 & 0.0 \\
\hline Bulldog & 13 & 7 & $53.8^{*}$ & 3 & 23.1 & 0 & 0.0 & 0 & 0.0 & 4 & $30.8 * *$ & 1 & 7.7 \\
\hline German Shepherd & 25 & 10 & 40.0 & 4 & 16.0 & 2 & 8.0 & 1 & 4.0 & 3 & 12.0 & 1 & 4.0 \\
\hline Golden retriever & 32 & 11 & 34.4 & 7 & 21.9 & 2 & 6.3 & 4 & 12.5 & 1 & 3.1 & 1 & 3.1 \\
\hline Mixed background & 35 & 12 & 34.3 & 4 & 11.4 & 4 & 11.4 & 1 & 2.9 & 2 & 5.7 & 5 & $14.3 * *$ \\
\hline Labrador retriever & 24 & 7 & 29.2 & 5 & 20.8 & 1 & 4.2 & 1 & 4.2 & 1 & 4.2 & 0 & 0.0 \\
\hline Chihuahua & 12 & 3 & 25.0 & 3 & 25.0 & 0 & 0.0 & 0 & 0.0 & 0 & 0.0 & 1 & 8.3 \\
\hline Siberian Husky & 25 & 6 & 24.0 & 2 & 8.0 & 1 & 4.0 & 1 & 4.0 & 1 & 4.0 & 1 & 4.0 \\
\hline Pembroke Welsh Corgi & 13 & 3 & 23.1 & 2 & 15.4 & 0 & 0.0 & 0 & 0.0 & 1 & 7.7 & 0 & 0.0 \\
\hline Samoyed & 18 & 4 & 22.2 & 2 & 11.1 & 2 & 11.1 & 1 & 5.6 & 0 & 0.0 & 0 & 0.0 \\
\hline Pomeranian & 21 & 4 & 19.0 & 2 & 9.5 & 1 & 4.8 & 2 & 9.5 & 1 & 4.8 & 0 & 0.0 \\
\hline Poodles & 142 & 25 & $17.6^{* *}$ & 13 & 9.2 & 3 & 2.1 & 9 & 6.3 & 1 & $0.7 * *$ & 0 & $0.0^{*}$ \\
\hline Chowchow & 13 & 1 & 7.7 & 0 & 0.0 & 1 & 7.7 & 0 & 0.0 & 0 & 0.0 & 0 & 0.0 \\
\hline
\end{tabular}

Only breeds with $\geq 12$ cases were included. Statistical significance was calculated by Fisher's exact test for individual breeds in reference to the sum of other breeds $(4 \times 4$; two-tailed). Numbers in italics indicate groups with infection rates that were statistically significantly different from other groups. $* p<0.05 ; * *<<0.01 ; * * * p<0.001$

vaccinated). However, while we were unable to gain details from the clients on the deworming drugs used for their dogs, the four deworming formulas commonly used by veterinarians in China were mainly labeled as anthelminthic, including Drontal Plus flavor (praziquantel/pyrantel pamoate/febantel) against helminths (nematodes and tapeworms); fenbendazole tablets against roundworms, whipworms, hookworms, and some tapeworms (but ineffective against Dipylidium caninum); and Heartguard Plus or Iverhart Plus (ivermection/pyrantel) against heartworm, ascarids and hookworms. On the other hand, most of the ingredients in these formulas were also experimentally shown to have efficacy against some protozoan parasites, such as Giardia spp. [e.g., (Barr et al. 1994; Bowman et al. 2009; Foletto et al. 2015; Giangaspero et al. 2002; Montoya et al. 2008; Moron-Soto et al. 2017; Zajac et al. 1998)], which might explain partially the benefits of these deworming formulas against protozoan infections. Additionally, many companion dogs might have received specific anti-protozoan treatments, but their owners simply considered all treatments as deworming.

No statistically significant associations were observed for other risk factors. It was noticeable that source of drinking water was not a significant risk factor (i.e., tap water vs. purified or boiled water; $\mathrm{OR}=0.8,1.1,1.1$ and $p=0.4328,0.6730,0.6423$, respectively) (Table 3), although both Giardia and Cryptosporidium are generally considered water-borne pathogens. This observation implies that direct contact between animals and/or humans, rather than the water, might be the major contributor for the fecal-to-oral transmission route of many parasites in household dogs at least for those in the Beijing region.

\section{Genotyping of $G$. duodenalis}

Using nested PCR targeting SSU rRNA genes, we observed that 53 specimens were positive for $G$. duodenalis. The identities of PCR products were confirmed by directly sequencing the PCR products. Among the Giardia-positive specimens, we have successfully amplified $g d h$ and/or $b g$ gene fragments from 21 specimens for subsequent genotyping analysis. Among them, 14 specimens produced PCR amplicons for both $g d h$ and $b g$ genes that were suitable for direct sequencing, while only one produced suitable amplicons for $g d h$ only, and six for $b g$. In the 14 specimens with sequences for both $g d h$ and $b g$ genes, the genotypes determined by one gene fully agreed with those determined by the other, suggesting that the genotypes for the other 7 specimens determined by one of the two genes were reasonably reliable. Accordingly, we assigned the genotypes of the 21 specimens based on $g d h$ and/or $b g$ genes and observed that assemblage $\mathrm{D}$ was the dominant genotype $(n=15,71.4 \%)$, followed by $\mathrm{C}(n=5,23.8 \%)$ and $\mathrm{F}(\mathrm{n}=1$, $4.8 \%$ ) (Table 4). We also attempted genotyping at the tpi locus, but could only obtain good-quality PCR amplicons from 4 specimens, all belonging to the assemblage $\mathrm{C}$ sequences that were in agreement with the typing based on $g d h$ and/or $b g$ loci (data not shown).

Assemblages C and D are mostly dog-specific (Feng and Xiao 2011; Heyworth 2016). However, it appears that this is the first time that the cat-specific assemblage $F$ was detected in 
Table 3 Risk factors analysis on the infection by enteric parasites in companion dogs

\begin{tabular}{|c|c|c|c|c|c|c|}
\hline Risk factor & Group & Total $n$ & Positive $n$ & Rate (\%) & Odds ratio $\left(\mathrm{Cl}_{95}\right)$ & $p$ value \\
\hline \multirow[t]{3}{*}{ Age } & $\leq 6$ months & 238 & 95 & 39.9 & $5.0(3.1-8.0)$ & $<0.0001 * * *$ \\
\hline & 6-12 months & 79 & 15 & 19.0 & $0.6(0.4-1.2)$ & 0.1601 \\
\hline & $\geq 12$ months & 168 & 14 & 8.3 & $0.2(0.1-0.3)$ & $<0.0001 * * *$ \\
\hline \multirow[t]{2}{*}{ Gender } & Male & 320 & 76 & 23.8 & $0.8(0.5-1.2)$ & 0.1891 \\
\hline & Female & 164 & 48 & 29.3 & $1.3(0.9-2.0)$ & 0.1891 \\
\hline \multirow[t]{4}{*}{ Origin } & Dog farms & 235 & 56 & 23.8 & $0.8(0.6-1.3)$ & 0.4067 \\
\hline & Pet stores & 108 & 32 & 29.6 & $1.3(0.8-2.1)$ & 0.3167 \\
\hline & Pet owners & 118 & 30 & 25.4 & $1.0(0.6-1.6)$ & 1.0000 \\
\hline & Street/others & 24 & 6 & 33.3 & $1.0(0.4-2.5)$ & 1.0000 \\
\hline \multirow[t]{4}{*}{ Season } & Mar-May & 127 & 39 & 30.7 & $1.4(0.9-2.2)$ & 0.1255 \\
\hline & Jun-Aug & 118 & 31 & 26.3 & $1.1(0.7-1.7)$ & 0.9035 \\
\hline & Sep-Nov & 101 & 27 & 26.7 & $1.1(0.7-1.8)$ & 0.7980 \\
\hline & Dec-Feb & 139 & 27 & 19.4 & $0.6(0.4-1.0)$ & 0.0511 \\
\hline \multirow[t]{2}{*}{ Live with other pets } & Yes & 108 & 31 & 28.7 & $1.2(0.8-2.0)$ & 0.3826 \\
\hline & No & 375 & 92 & 24.5 & $0.8(0.5-1.3)$ & 0.3826 \\
\hline \multirow[t]{3}{*}{ Food source } & Commercial & 374 & 93 & 24.8 & $0.9(0.6-1.4)$ & 0.6195 \\
\hline & Self-prepared & 48 & 16 & 33.3 & $1.5(0.8-2.9)$ & 0.2205 \\
\hline & Mixed & 62 & 14 & 22.6 & $0.8(0.4-1.6)$ & 0.6421 \\
\hline \multirow[t]{3}{*}{ Water source } & Tape water & 152 & 35 & 23.0 & $0.8(0.5-1.3)$ & 0.4328 \\
\hline & Purified water & 199 & 53 & 26.6 & $1.1(0.7-1.7)$ & 0.6730 \\
\hline & Boiled water & 133 & 36 & 27.1 & $1.1(0.7-1.8)$ & 0.6423 \\
\hline \multirow[t]{2}{*}{ Vaccination } & Yes & 293 & 61 & 20.8 & $0.5(0.4-0.8)$ & $0.0040 * *$ \\
\hline & No & 192 & 63 & 32.8 & $1.9(1.2-2.8)$ & $0.0040 * *$ \\
\hline \multirow[t]{2}{*}{ Deworming (overall) } & Yes & 264 & 49 & 18.6 & $0.4(0.3-0.7)$ & $0.0002 * * *$ \\
\hline & No & 220 & 74 & 33.6 & $2.2(1.5-3.4)$ & $0.0002 * * *$ \\
\hline \multirow[t]{2}{*}{ Deworming (protozoa) } & Yes & 264 & 36 & 13.6 & $0.4(0.3-0.7)$ & $0.0003 * * *$ \\
\hline & No & 220 & 59 & 26.8 & $2.3(1.5-3.7)$ & $0.0003 * * *$ \\
\hline \multirow[t]{2}{*}{ Deworming (helminths) } & Yes & 264 & 17 & 6.4 & $0.6(0.3-1.1)$ & 0.1355 \\
\hline & No & 220 & 23 & 10.5 & $1.7(0.9-3.3)$ & 0.1355 \\
\hline
\end{tabular}

${ }^{\mathrm{a}}$ For each risk factor, the odds ratio for the specified group was calculated in reference to the other group or the sum of all other groups in the presence of only two groups or more than two groups, respectively. The $95 \%$ confidence intervals $\left(\mathrm{CI}_{95}\right)$ for the odds ratios were calculated using Woolf method. $P$ values were calculated by Fisher's exact test

$* p<0.05 ; * * p<0.01 ; * * * p<0.001$

a dog, supported by genotyping analysis based on both $g d h$ (KY753404) and $b g$ (KY753419) gene sequences. It is less likely that the specimen was contaminated with cat feces, as individual fecal samples were freshly collected from dogs in the hospital, the positive control used DNA isolated from pooled dog feces, and the assay on this specimen was performed multiple times. Although less common, assemblage $\mathrm{F}$ was previously reported in pigs and cetaceans among others (Feng and Xiao 2011; Heyworth 2016), which implies that a small number of assemblage $F$ strains might be present in animals other than cats or capable of transmitting from cats to other animals to cause ephemeral infection.
Table 4 Summary of the assemblages of Giardia duodenalis based on genotyping at $g d h$ and $b g$ loci

\begin{tabular}{lllll}
\hline Giardia duodenalis assemblage & By $b g$ only & By $g d h$ only & By both $b g \& g d h$ & Total $N(\%)$ \\
\hline Assemblage C & 1 & 0 & 4 & $5(23.8 \%)$ \\
Assemblage D & 5 & 1 & 9 & $15(71.4 \%)$ \\
Assemblage F & 0 & 0 & 1 & $1(4.8 \%)$ \\
Grand total $N(\%)$ & & & & $21(100 \%)$ \\
\hline
\end{tabular}




\section{Molecular detection of Cryptosporidium spp.}

Based on SSU rRNA gene sequences, only $C$. canis $(n=20)$ was detected (Table 4). There were two type of SSU rRNA sequences that differed from each other by a single nucleotide polymorphism (SNP) as represented by sequences KX858906 and KX858907.

\section{Implication on public health}

Companion animals including dogs are one of the important One Health components, particularly in transmitting zoonotic diseases. Among the two common trichomonads $(n=21$ in this study), Pentatrichomonas hominis is zoonotic and more common in dogs in China and many other regions (Grellet et al. 2013; Li et al. 2016; Tolbert et al. 2012). However, $P$. hominis is generally considered nonpathogenic to humans (Gookin et al. 2007; Meloni et al. 2011). Tritrichomonas foetus is less common in dogs and not considered a human pathogen, although it may cause genitourinary infection in cattle and diarrhea in cats and dogs (Gookin et al. 2005; Michi et al. 2016; Tolbert and Gookin 2016). However, a rare case of $T$. foetus peritonitis was reported in an immunodeficient patient (Lacassin 2005), implying its potential to cause opportunistic infection in humans. Among dog ascarids, Toxocara canis is the most common parasite causing toxocariasis in humans. In this survey, we observed 17 cases of $T$. canis among 485 dogs with diarrhea, suggesting that companion dogs may serve as a source for transmitting this roundworm to humans.

Among the other protozoan parasites in dogs, Cystoisopora species $(n=21)$ are non-human pathogens, whereas some genotypes/species of Giardia and Cryptosporidium are zoonotic. For $G$. duodenalis, humans are infected mostly by assemblages A and B isolates, but cases of human infections by assemblages C, D, and F isolates were also reported in Europe and Thailand (Feng and Xiao 2011). For Cryptosporidium species, $C$. canis may also infect humans, particularly in immunocompromised individuals (Xiao et al. 2007).

In summary, this study indicates that household dogs may be a source, although not a major source, of zoonotic transmission of some parasitic infections in humans in the Beijing region. Practice of careful sanitation is recommended for the owners and family members of companion dogs, particularly those with weak or weakened immunity such as infants, elderlies, and patients with immunosuppression.

Acknowledgements The authors gratefully thank all veterinaries working in the Clinic Laboratory in CAU teaching veterinary hospital and pet owners who participated in this study. This study was supported by grants from the National Innovation Experiment Program for Undergraduates of China (No. 201510019073) and Chinese Universities Scientific Fund for Postgraduates (No. 2015DY003).

\section{References}

Abe N, Sawano Y, Yamada K, Kimata I, Iseki M (2002) Cryptosporidium infection in dogs in Osaka, Japan. Vet Parasitol 108:185-193

Adamu H, Petros B, Zhang G, Kassa H, Amer S, Ye J, Feng Y, Xiao L (2014) Distribution and clinical manifestations of Cryptosporidium species and subtypes in HIV/AIDS patients in Ethiopia. PLoS Negl Trop Dis 8:e2831. https://doi.org/10.1371/journal.pntd.0002831

Baneth G, Thamsborg SM, Otranto D, Guillot J, Blaga R, Deplazes P, Solano-Gallego L (2016) Major parasitic zoonoses associated with dogs and cats in Europe. J Comp Pathol 155:S54-S74. https://doi. org/10.1016/j.jcpa.2015.10.179

Barr SC, Bowman DD, Heller RL (1994) Efficacy of fenbendazole against giardiasis in dogs. Am J Vet Res 55:988-990

Bowman DD, Liotta JL, Ulrich M, Charles SD, Heine J, Schaper R (2009) Treatment of naturally occurring, asymptomatic Giardia sp. in dogs with Drontal Plus flavour tablets. Parasitol Res 105(Suppl 1):S125-S134. https://doi.org/10.1007/s00436-009-1503-0

Cama VA, Ross JM, Crawford S, Kawai V, Chavez-Valdez R, Vargas D, Vivar A, Ticona E, Navincopa M, Williamson J (2007) Differences in clinical manifestations among Cryptosporidium species and subtypes in HIV-infected persons. J Infect Dis 196:684-691

Denholm KM, Haitjema H, Gwynne BJ, Morgan UM, Irwin PJ (2001) Concurrent Cryptosporidium and parvovirus infections in a puppy. Aust Vet J 79:98-101

Feng Y, Xiao L (2011) Zoonotic potential and molecular epidemiology of Giardia species and giardiasis. Clin Microbiol Rev 24:110-140. https://doi.org/10.1128/CMR.00033-10

Foletto VR, Vanz F, Gazarini L, Stern CA, Tonussi CR (2015) Efficacy and security of ivermectin given orally to rats naturally infected with Syphacia spp., Giardia spp. and Hymenolepis nana. Lab Anim 49: 196-200. https://doi.org/10.1177/0023677214562850

Giangaspero A, Traldi G, Paoletti B, Traversa D, Bianciardi P (2002) Efficacy of pyrantel embonate, febantel and praziquantel against Giardia species in naturally infected adult dogs. Vet Rec 150:184 186

Gookin JL, Birkenheuer AJ, St John V, Spector M, Levy MG (2005) Molecular characterization of trichomonads from feces of dogs with diarrhea. J Parasitol 91:939-943. https://doi.org/10.1645/GE-474R.

Gookin JL, Stauffer SH, Levy MG (2007) Identification of Pentatrichomonas hominis in feline fecal samples by polymerase chain reaction assay. Vet Parasitol 145:11-15. https://doi.org/10. 1016/j.vetpar.2006.10.020

Gracio AJ, Richter J, Komnenou AT, Gracio MA (2015) Onchocerciasis caused by Onchocerca lupi: an emerging zoonotic infection. Systematic review. Parasitol Res 114:2401-2413. https://doi.org/ 10.1007/s00436-015-4535-7

Grellet A, Brunopolack FA, Boucraut-Baralon C, Grandjean D, Vandewynckel L, Cian A, Meloni D, Viscogliosi E (2013) Prevalence, risk factors of infection and molecular characterization of trichomonads in puppies from French breeding kennels. Vet Parasitol 197:418-426. https://doi.org/10.1016/j.vetpar.2013.07. 030

Grinnage-Pulley T, Scott B, Petersen CA (2016) A mother's gift: congenital transmission of Trypanosoma and Leishmania species. PLoS Pathog 12:e1005302. https://doi.org/10.1371/journal.ppat.1005302

Heyworth MF (2016) Giardia duodenalis genetic assemblages and hosts. Parasite 23:13. https://doi.org/10.1051/parasite/2016013

Lacassin F (2005) Rare case of trichomonal peritonitis. Infect Dis 11: $1607-1609$

Lalle M, Pozio E, Capelli G, Bruschi F, Crotti D, Caccio SM (2005) Genetic heterogeneity at the beta-giardin locus among human and animal isolates of Giardia duodenalis and identification of 
potentially zoonotic subgenotypes. Int J Parasitol 35:207-213. https://doi.org/10.1016/j.ijpara.2004.10.022

Li WC, Wang K, Zhang W, Wu J, Gu YF, Zhang XC (2016) Prevalence and molecular characterization of intestinal Trichomonads in pet dogs in East China. Korean J Parasitol 54:703-710. https://doi.org/ 10.3347/kjp.2016.54.6.703

Matchock RL (2015) Pet ownership and physical health. Curr Opin Psychiatry 28:386-392. https://doi.org/10.1097/YCO. 0000000000000183

Meloni D, Mantini C, Goustille J, Desoubeaux G, Maakaroun-Vermesse Z, Chandenier J, Gantois N, Duboucher C, Fiori PL, Dei-Cas E, Duong TH, Viscogliosi E (2011) Molecular identification of Pentatrichomonas hominis in two patients with gastrointestinal symptoms. J Clin Pathol 64:933-935. https://doi.org/10.1136/jcp. 2011.089326

Michi AN, Favetto PH, Kastelic J, Cobo ER (2016) A review of sexually transmitted bovine trichomoniasis and campylobacteriosis affecting cattle reproductive health. Theriogenology 85:781-791. https://doi. org/10.1016/j.theriogenology.2015.10.037

Montoya A, Dado D, Mateo M, Espinosa C, Miro G (2008) Efficacy of Drontal Flavour Plus (50 mg praziquantel, $144 \mathrm{mg}$ pyrantel embonate, $150 \mathrm{mg}$ febantel per tablet) against Giardia sp in naturally infected dogs. Parasitol Res 103:1141-1144. https://doi.org/10. 1007/s00436-008-1107-0

Moron-Soto M, Gutierrez L, Sumano H, Tapia G, Alcala-Canto Y (2017) Efficacy of nitazoxanide to treat natural Giardia infections in dogs. Parasit Vectors 10:52. https://doi.org/10.1186/s13071-017-1998-7

Petersen C (1992) Cryptosporidiosis in patients infected with the human immunodeficiency virus. Clin Infect Dis 15:903-909

Qi M, Zhou H, Wang H, Wang R, Xiao L, Arrowood MJ, Li J, Zhang L (2015) Molecular identification of Cryptosporidium spp. and Giardia duodenalis in grazing horses from Xinjiang, China. Vet Parasitol 209:169-172. https://doi.org/10.1016/j.vetpar.2015.02. 030
Read CM, Monis PT, Thompson RC (2004) Discrimination of all genotypes of Giardia duodenalis at the glutamate dehydrogenase locus using PCR-RFLP. Infect Genet Evol 4:125-130. https://doi.org/10. 1016/j.meegid.2004.02.001

Robertson ID, Thompson RC (2002) Enteric parasitic zoonoses of domesticated dogs and cats. Microbes Infect 4:867-873

Takashima GK, Day MJ (2014) Setting the One Health agenda and the human-companion animal bond. Int J Environ Res Public Health 11: 11110-11120. https://doi.org/10.3390/ijerph111111110

Tasic-Otasevic SA, Trenkic Bozinovic MS, Gabrielli SV, Genchi C (2015) Canine and human Dirofilaria infections in the Balkan Peninsula. Vet Parasitol 209:151-156. https://doi.org/10.1016/j. vetpar.2015.02.016

Tolbert MK, Gookin JL (2016) Mechanisms of Tritrichomonas foetus Pathogenicity in Cats with Insights from Venereal Trichomonosis. J Vet Intern Med 30:516-526. https://doi.org/10.1111/jvim. 13920

Tolbert MK, Leutenegger CM, Lobetti R, Birrell J, Gookin JL (2012) Species identification of trichomonads and associated coinfections in dogs with diarrhea and suspected trichomonosis. Vet Parasitol 187:319-322. https://doi.org/10.1016/j.vetpar.2011.12.031

Wang H, Zhao G, Chen G, Jian F, Zhang S, Feng C, Wang R, Zhu J, Dong H, Hua J, Wang M, Zhang L (2014) Multilocus genotyping of Giardia duodenalis in dairy cattle in Henan, China. PLoS One 9: e100453. https://doi.org/10.1371/journal.pone.0100453

Xiao L, Cama VA, Cabrera L, Ortega Y, Pearson J, Gilman RH (2007) Possible transmission of Cryptosporidium canis among children and a dog in a household. J Clin Microbiol 45:2014-2016. https://doi. org/10.1128/JCM.00503-07

Xiao L, Morgan UM, Limor J, Escalante A, Arrowood M, Shulaw W, Thompson RCA, Fayer R, Lal AA (1999) Genetic diversity within Cryptosporidium parvum and related Cryptosporidium species. Appl Environ Microbiol 65:3386-3391

Zajac AM, LaBranche TP, Donoghue AR, Chu TC (1998) Efficacy of fenbendazole in the treatment of experimental Giardia infection in dogs. Am J Vet Res 59:61-63 